\title{
The AHA Guidelines for the Management of SAH: What We Know and So Much We Need to Learn
}

\author{
Alejandro A. Rabinstein
}

Published online: 24 April 2009

(C) Humana Press Inc. 2009

Bederson JB, Connolly ES Jr., Batjer HH, Dacey RG, Dion JE, Diringer MN, Duldner JE Jr., Harbaugh RE, Patel AB, Rosenwasser RH. Guidelines for the management of aneurysmal subarachnoid hemorrhage: a statement for healthcare professionals from a special writing group of the Stroke Council, American Heart Association. Stroke. 2009;40:994-1025.

The publication of the update of the AHA Guidelines for the Management of Aneurysmal Subarachnoid Hemorrhage (SAH) had been long awaited. The previous version of the guidelines dated back to 1994 [1]. Hence, the writing group had to take on the colossal task of reviewing the literature on SAH spanning from 1994 to 2006. It is no surprise that the resulting document is long and boasts over 500 references. These guidelines are a great resource to review what we know about aneurysmal SAH and an even better framework to discuss and highlight what we still need to learn to improve the care of SAH patients.

The purpose of this succinct comment on the AHA SAH guidelines will not be to criticize its deficiencies. The authors should actually be commended for their courage and dedication. Writing these guidelines must have been a tremendously challenging and time-demanding chore. Instead, I will try to concentrate on the main messages of the document and point out the gaps on our knowledge which become readily apparent after reading the guidelines and should be the focus of future research.

Guidelines must be based on a careful review of the evidence. However, there are few areas in the management of SAH for which we have conclusive evidence from

A. A. Rabinstein $(\bowtie)$

Mayo Clinic, 200 First Street SW, Rochester, MN 55905, USA

e-mail: rabinstein.alejandro@mayo.edu rigorous randomized, controlled clinical trials, or large prospective cohort studies. In the absence of level of evidence A (i.e., data from multiple randomized trials for treatment or from multiple prospective cohort studies using a reference standard applied by a masked evaluator), the recommendations of the authors need to be cautious. If the questions are not clearly resolved by undeniable evidence, different interpretations of lesser quality studies and the positions of experts on the field have to be weighed in proposing those recommendations. Satisfying all of those positions, which are often encountered albeit based on reasonable arguments, may be extremely difficult. This caveat accounts for the paucity of Class I recommendations and may explain why the writing group chose a relatively open language in the wording of various recommendations (for example when referring to coiling versus clipping of the ruptured aneurysm, the use of intraoperative hypothermia during aneurysm clipping, or the early, short-term administration of antifibrinolytics in selected patients at high risk of rebleeding).

Despite its length, these guidelines cannot be fully comprehensive. A whole book would be required to address all the topics that pertain to the management of aneurysmal SAH patients in detail. But even with that consideration in mind, it is notable to realize that the document is clearly more solid when discussing the epidemiology of SAH than it is when referring to the management of vasospasm, despite the fact that the timely diagnosis and effective treatment of vasospasm constitutes one of the most important goals in the neurocritical care of these patients. The reason for this discrepancy is simple. The literature on the epidemiology of SAH is far stronger than the information on the diagnosis and treatment of vasospasm. The guidelines merely reflect the deficiencies in our scientific knowledge. 
The following two sections will summarize the most important recommendations provided in the guidelines based on available evidence and outline some of the areas in which quality information is lacking and further research is needed.

\section{The Present Evidence}

\section{Risk Factors for Aneurysmal SAH}

Hypertension, smoking, heavy alcohol use, consumption of sympathomimetic drugs (such as cocaine), certain inherited conditions (autosomal dominant polycystic kidney disease, Ehlers Danlos type IV), and familial intracranial aneurysms are associated with increased risk of SAH. Also, patients with a previous ruptured aneurysm who have other aneurysms may be at increased risk.

\section{Prevention of SAH}

Treatment of hypertension and smoking cessation are reasonable but their value in reducing the risk of SAH has not been determined. Screening individuals at high-risk for harboring intracranial aneurysms is given a Class IIb recommendation (i.e., usefulness less well-established by evidence or opinion), but there is very limited data to evaluate if screening these individuals is cost-effective and to assess the risk-benefit ratio of treating those individuals with documented aneurysms.

\section{Natural History and Outcome}

It is clear that the severity of the hemorrhage as gauged by the initial clinical presentation is the most important determinant of outcome. Patients suspected of having $\mathrm{SAH}$ must be evaluated emergently because the risk of rebleeding is highest in the first few hours after aneurysm rupture $[2,3]$.

\section{Diagnosis of SAH}

SAH is too often misdiagnosed [4]. Head CT scan must always be done when SAH is part of the differential diagnosis and lumbar puncture should follow when the CT scan is negative. Catheter cerebral angiography should be performed in patients with SAH (Class I, Level of Evidence B). Non-invasive vascular imaging techniques (MR angiography or CT angiography) can be considered when conventional angiography cannot be performed in a timely manner (Class IIb, Level of Evidence B). These non-invasive techniques are quite sensitive for the detection of aneurysms $\geq 5 \mathrm{~mm}$ in maximal diameter, but their sensitivity remains lower (albeit improving) for smaller aneurysms.

\section{Emergency Evaluation and Preoperative Care}

Bedrest is useful but certainly not sufficient to prevent rebleeding. Blood pressure should be monitored and controlled balancing the possible risk of hypertension inducing rebleeding versus hypotension compromising cerebral perfusion. Short-term use of antifibrinolytics started very promptly after aneurysm rupture "may be reasonable" in selected patients deemed to be at particularly high risk of rebleeding, as long as this practice is combined with early treatment of the aneurysm and measures to prevent volume contraction and hypotension.

\section{Systems of Care}

Early referral to high-volume centers with access to cerebrovascular surgeons and endovascular specialists is recommended (Class IIa, Level of Evidence B) [5-8].

\section{Treatment of the Ruptured Aneurysm}

This was the longest section of the guidelines and tried to balance the results of ISAT (the only international, multicenter, prospective, randomized study of endovascular versus surgical treatment of ruptured aneurysms) [9, 10] against other relevant studies. The writing group reasonably concluded that surgical clipping or endovascular coiling need to be performed to secure the ruptured aneurysm to prevent rebleeding and pointed out that complete obliteration of the aneurysm should be the goal. Wrapped, coated, and incompletely coiled or clipped aneurysms have persistent risk of recurrent rupture. Early aneurysm treatment should be pursued, at least in the majority of cases.

Regarding the relative merits of coiling versus clipping the recommendation reads: "For patients with ruptured aneurysms judged by an experienced team of cerebrovascular surgeons and endovascular practitioners to be technically amenable to endovascular coiling and neurosurgical clipping, endovascular coiling could be beneficial (Class I, Level of Evidence B)." In other words, when either procedure is feasible, coiling may be preferred. However, the writing group emphasized the importance of considering individual characteristics and reaching the decision by consensus. Although this was not specifically mentioned in the recommendations per se, the question of the durability of coiling and the delayed risk of recurrent hemorrhage after incomplete coiling is discussed in detail in the text of the guidelines, and careful angiographic monitoring of coiled aneurysms is strongly advised. 
Anesthetic Management

The main recommendation is to minimize the degree and duration of intraoperative hypotension. Although the practices of induced hypertension during vessel clamping and intraoperative hypothermia have not been proven valuable by available evidence, they are presented as reasonable options in some cases (albeit as Class IIb and III recommendations, respectively).

\section{Management of Vasospasm}

Oral nimodipine is recommended based on level of evidence A indicating improved functional outcomes in patients treated with this calcium channel blocker; yet, the writing group recognizes that the mechanism responsible for the modest benefit is unclear. The rest of the recommendations are presented using a language that denotes the problems generated by the profound gaps between evidence and practice. Maintaining euvolemia and avoiding hypovolemia are "probably indicated," triple $\mathrm{H}$ therapy is a "reasonable approach" to treat symptomatic vasospasm, and angioplasty and super-selective intra-arterial infusion of vasodilators is also "reasonable" as an alternative therapy. Needless to say, all these practices are considered standard of care by most of us treating patients with vasospasm from SAH [11, 12]. However, there is remarkably little rigorous scientific data supporting their use.

\section{Management of Hydrocephalus}

Temporary or permanent CSF diversion is recommended for patients with symptomatic hydrocephalus (Class I, Level of Evidence B). In patients with ventriculomegaly and diminished level of consciousness ventriculostomy "may be beneficial."

\section{Management of Seizures}

The guidelines state that the administration of prophylactic anticonvulsants may be considered in the immediate posthemorrhagic period (Class IIb), although acknowledging that there is very little data to support this practice. Longterm use of anticonvulsants is discouraged, except in selected cases. A study indicating deleterious effects from the use of phenytoin is presented in the text of the guidelines [13].

\section{Management of Hyponatremia}

The guidelines underscore that hyponatremia is often associated with volume contraction and recommend avoiding administration of large volumes of hypotonic fluids and hypovolemia (Class I, Level of Evidence B) [14]. Fludrocortisone and hypertonic saline are presented as reasonable treatment options.

\section{Future Research Needed}

As I was reading the guidelines, I started writing down various unresolved questions which I believe should be addressed by future research. I am sure this is only a partial list but it will hopefully serve to illustrate the many aspects of SAH management in which further progress needs to occur.

- Who should be screened for intracranial aneurysms and how?

- What are the mechanisms of acute brain injury at the time of SAH?

- What would be the most important variables to be incorporated in a tool designed to help paramedics and Emergency Department personnel recognize SAH?

- What constitutes the best emergent care of patients with SAH? For example, what should be the target blood pressure?

- Should SAH care be regionalized?

- What is the true value of newer endovascular techniques (e.g., stent-assisted coiling, bioactive coils)?

- What is the best protocol to monitor coiled aneurysms (how often should angiograms be repeated? for how long? using what imaging technique?)

- Is intraoperative hypothermia actually valuable in certain cases? How about intraoperative induced hypertension?

- What is the added value of caring for these patients in a neurological ICU and what are the practices which afford greater benefit?

- Is outcome improved by using standardized protocols for SAH care?

- What is the value of invasive multi-modality brain monitoring in poor-grade patients?

- What is the role of newer diagnostic techniques (e.g., CT perfusion, vasoreactivity studies, brain tissue oxygen probes) for the timely detection of vasospasm and cerebral hypoperfusion?

- What is the value of novel treatment strategies for the prevention of delayed ischemia (e.g., endothelin antagonists, statins, magnesium, lumbar drainage, prophylactic angioplasty, nitric oxide donors)?

- What is the best way to implement hemodynamic augmentation?

- What is the value of albumin in SAH?

- Should poor-grade patients be kept hypothermic? 
- What other mechanisms in addition to vasospasm cause secondary brain damage after SAH?

- Is profound anemia worse than transfusions?

- Are anticonvulsants useful if one avoids phenytoin?

- What is the best timing and method for discontinuation of ventricular drainage in patients with early hydrocephalus?

- What is the best functional outcome endpoint for future studies? Are there reliable surrogate endpoints? Should we standardize functional outcome assessment tools across SAH studies?

These questions and many others demand answers. Hard cooperative work and increased funding for research in SAH will be necessary to acquire them.

\section{References}

1. Mayberg MR, Batjer HH, Dacey R, Diringer M, Haley EC, Heros $\mathrm{RC}$, et al. Guidelines for the management of aneurysmal subarachnoid hemorrhage. A statement for healthcare professionals from a special writing group of the Stroke Council, American Heart Association. Stroke. 1994;25:2315-28.

2. Kassell NF, Torner JC. Aneurysmal rebleeding: a preliminary report from the Cooperative Aneurysm Study. Neurosurgery. 1983;13:479-81.

3. Ohkuma H, Tsurutani H, Suzuki S. Incidence and significance of early aneurysmal rebleeding before neurosurgical or neurological management. Stroke. 2001;32:1176-80.

4. Kowalski RG, Claassen J, Kreiter KT, Bates JE, Ostapkovich ND, Connolly ES, et al. Initial misdiagnosis and outcome after subarachnoid hemorrhage. JAMA. 2004;291:866-9. doi:10.1001/ jama.291.7.866.

5. Cross DTIII, Tirschwell DL, Clark MA, Tuden D, Derdeyn CP, Moran CJ, et al. Mortality rates after subarachnoid hemorrhage: variations according to hospital case volume in 18 states. J Neurosurg. 2003;99:810-7.
6. Johnston SC. Effect of endovascular services and hospital volume on cerebral aneurysm treatment outcomes. Stroke. 2000;31:111-7.

7. Berman MF, Solomon RA, Mayer SA, Johnston SC, Yung PP. Impact of hospital-related factors on outcome after treatment of cerebral aneurysms. Stroke. 2003;34:2200-7. doi:10.1161/01. STR.0000086528.32334.06.

8. Bardach NS, Zhao S, Gress DR, Lawton MT, Johnston SC. Association between subarachnoid hemorrhage outcomes and number of cases treated at California hospitals. Stroke. 2002;33:1851-6.

9. Molyneux A, Kerr R, Stratton I, Sandercock P, Clarke M, Shrimpton J, et al. International Subarachnoid Aneurysm Trial (ISAT) of neurosurgical clipping versus endovascular coiling in 2143 patients with ruptured intracranial aneurysms: a randomised trial. Lancet. 2002;360:1267-74. doi:10.1016/S0140-6736(02) 11314-6.

10. Molyneux AJ, Kerr RS, Yu LM, Clarke M, Sneade M, Yarnold $\mathrm{JA}$, et al. International subarachnoid aneurysm trial (ISAT) of neurosurgical clipping versus endovascular coiling in 2143 patients with ruptured intracranial aneurysms: a randomised comparison of effects on survival, dependency, seizures, rebleeding, subgroups, and aneurysm occlusion. Lancet. 2005;366:80917. doi:10.1016/S0140-6736(05)67214-5.

11. Rabinstein AA, Wijdicks EF. Cerebral vasospasm in subarachnoid hemorrhage. Curr Treat Options Neurol. 2005;7:99-107. doi:10.1007/s11940-005-0019-x.

12. Sen J, Belli A, Albon H, Morgan L, Petzold A, Kitchen N. Triple$\mathrm{H}$ therapy in the management of aneurysmal subarachnoid haemorrhage. Lancet Neurol. 2003;2:614-21. doi:10.1016/ S1474-4422(03)00531-3.

13. Naidech AM, Kreiter KT, Janjua N, Ostapkovich N, Parra A, Commichau $\mathrm{C}$, et al. Phenytoin exposure is associated with functional and cognitive disability after subarachnoid hemorrhage. Stroke. 2005;36:583-7. doi:10.1161/01.STR.00001419 36.36596.1e.

14. Wijdicks EF, Vermeulen M, ten Haaf JA, Hijdra A, Bakker WH, van Gijn J. Volume depletion and natriuresis in patients with a ruptured intracranial aneurysm. Ann Neurol. 1985;18:211-6. doi: 10.1002/ana.410180208. 